\title{
LE FANTASTIQUE CLINIQUE DANS LES RÉCITS DE L'ÉTRANGETÉ PSYCHIQUE DE GUY DE MAUPASSANT
}

\begin{abstract}
Gadomska Katarzyna, Le fantastique clinique dans les récits de l'étrangeté psychique de Guy de Maupassant [Clinic fantastic literature in the short stories of Guy de Maupassant], Studia Romanica Posnaniensia, Adam Mickiewicz University Press, Poznań, vol. XXVII: 2001, pp. 11-23. ISBN 83-232-1039-X, ISSN 0137-2475.
\end{abstract}

The present work is a study of the clinic fantastic literature in the short stories by one of the most famous 19 th century authors Guy de Maupassant. In 19th century the tendency to reject the traditional fantastic literature consisting in unexpected and sudden interference of the supernatural powers into the real world.

Selon L.Vax «les sentiments sur lesquels joue l'émotion fantastique sont des sentiments esthétiquement négatifs: peur, horreur, dégoût»»; nous y ajouterions encore la folie. Au XIX ${ }^{\mathfrak{e}}$ siècle le fantastique clinique ${ }^{2}$, est très en vogue. On laisse de côté le fantastique traditionnel où les manifestations du surnaturel sont toujours extérieurs et qui met l'accent sur la réalité du phénomène. Le fantastique clinique, au contraire, se distingue par l'intériorisation de l'expérience insolite. Les angoisses du héros sont dans la première phase intériorisées pour réapparaitre plus tard à l'extérieur sur le mode hallucinatoire. Les visions, les fantasmes, les hallucinations se substituent alors au phénomène surnaturel traditionnel. Cependant la folie du héros n'est que suggérée, elle est une des interprétations possibles du récit. L'ambiguité ainsi créée permet de maintenir l'hésitation entre deux interprétations jusqu'à la fin de l'histoire. Les écrivains soucieux de l'effet fantastique n'éclairent presque jamais le mystère final. On peut se demander pourquoi c'est la folie qui est au centre du fantastique du $\mathrm{XIX}^{\mathrm{e}}$ siècle. Il ne faut pas oublier que malgré l'essor de

' L. V ax, La séduction de l'étrangeté, P.U.F., Paris 1987, p. 244.

${ }^{2}$ a. Pour éviter la confusion nous admettons que les termes: le fantastique clinique, le fantastique psychique, le fantastique d'ordre psychique sont equivalents; b. C'est J.-L. Bernard qui dans Dictionnaire de l'insolite et du fantastique utilise le terme «fantastique clinique» (Éditions du Dauphin, Paris 1977, p.119). 
la psychiatrie au $\mathrm{XIX}^{\mathrm{e}}$ siècle, l'esprit humain est toujours mystérieux, sa toute-puissance horrifie, il est le siège et la source de l'insolite. La folie favorise l'intrusion du surnaturel dans le récit et, en même temps, en tant qu'objet de recherches scientifiques, apporte la caution, de la vraisemblance au récit. La folie est souvent présentée comme un état suprême, un don qui permet de découvrir et de comprendre les mystères de l'univers. La folie dans les récits fantastiques peut aussi revêtir un autre aspect: elle est souvent la projection des angoisses et des obsessions du héros qui, anonyme et solitaire lutte en vain contre les manifestations de l'Autre. Le protagoniste, en marge de la société inđifférente, seul face au monde hostile, devient, en exprimant toutes ses hantises et toutes ses peurs, le porte - parole de la génération de fin de siècle. L'esprit humain est le domaine le plus exploré dans le fantastique psychologique, les thèmes fantastiques traditionnels, s'ils sont présents, revêtent une forme nouvelle, plus moderne, fondée sur la mise en évidence de l'étrangeté psychique du héros. Les tendances sont surtout visibles dans le fantastique de G. de Maupassant. Une très grande part de l'œuvre de Maupassant est consacrée à présenter la vision du monde d'un malade mental. Parmi les récits fantastiques de Maupassant on trouve plusieurs récits fantastiques de l'étrangeté psychique $^{3}$. Les récits de l'étrangeté psychique exposent des cas d'obsession, d'hallucination et de dédoublement. C'est l'univers mental du héros qui compte le plus. Le cerveau du malade devient un lieu hanté non par les démons mais par les cauchemars, les hantises et les fantasmes du héros. La folie est souvent présentée comme un autre état de conscience, non comme le mal, mais un état suprême qui permet d'être heureux, de voir et comprendre les choses inconnues par les autres. C'est une porte vers un ailleurs, vers un autre monde, peut-être meilleur. P.-J. Castex remarque: «[...] la folie devient le privilège d'esprits supérieurs, qui transfigurent, grâce à leurs lumières intérieures, les mornes données de la connaissance commune» ${ }^{4}$. Le plus souvent Maupassant décrit la folie dans sa phase ultime. Avec toute conscience il abandonne les premiers symptômes de la folie pour la montrer encore plus mystérieuse et plus inquiétante. La folie présentée par Maupassant n'a pas d'antécédents tels que l'hérédité ou l'alcool. Elle est angoissante car ce n'est pas une maladie que l'on peut essayer de comprendre et de soigner. P.-J. Castex dans Le conte fantastique en France de Nodier à Maupassant expose la thèse qu'il y a des traces autobiographiques dans des récits de Maupassant. Selon Castex, Maupassant touché d'une maladie mentale, a pris un vif intérêt pour la folie. L'écrivain fait les premières allusions à la démence du héros dans son premier récit fantastique La main d'écorché (1875) qui deviendra en 1883 La Main. Le narrateur de La Main M. Bermutier raconte à ses amis une affaire

${ }^{3}$ C'est G. Ponnau qui dans son étude La folie dans la littérature fantastique fait la distinction entre les trois types de récits fantastiques qui présentent les cas de démence, à côté de récits de l'étrangeté psychique, il énumère les histoires renouvelées de rèves, de paradis artificiels, de visions el les récits parapsychologiques; G. Pon nau, La folie dans la littérature fantastique, CNRS, Paris 1987, pp. $165-170$.

${ }^{4}$ P.-J. Castex, Le conte fantastique en France de Nodier à Maupassant, Corti, Paris 1994, p. 76. 
obscure qu'il a eu à suivre en tant que juge d'instruction à Ajaccio. Un jour sir John Rowell, l'homme fort mystérieux, y a loué une villa pour plusieurs années. Une fois pendant une soirée chez sir John M. Bermutier a remarqué une chose étrange: sur un carré de velours rouge était posée une main humaine desséchée, avec une énorme chaîne que l'attachait au mur. Sir John a expliqué que c'était la main de son ennemi. Un an plus tard Rowell a été étranglé, on a trouvé la chaîne brisée, la main n'y était plus. Un des doigts de cette main disparue a été retrouvé dans la bouche de l'Anglais. On n'a jamais retrouvé le coupable. Trois mois après ce crime odieux, une nuit, $M$. Bermutier avait un affreux cauchemar. Il a vu la main courir le long de ses murs. Le lendemain on l'a trouvée dans le cimetière sur la tombe de sir John Rowell. L'interprétation qui s'impose d'abord au lecteur est surnaturelle: c'est la main qui a tué sir John. Le motif d'une partie séparée du corps humain - qui tue, qui est nuisible, dangereuse - est bien connu dans la littérature fantastique. L.Vax dans La séduction de l'étrange écrit ainsi: «[...] une main coupée est une partie du corps humain qui semble hanter confusément la vie qui s'en est retirée. Organe qui saisit, empoigne, étrangle, pourquoi ne garderait - elle pas séparée du cerveau qui la dirigeait, la capacité d'agir autonome d'une <bête à cinq doigts $>$ ? $\rangle^{5}$. Après avoir examiné le corps le médecin constate que Rowell semble être étranglé par un squelette. M. Bermutier, au cours de l'enquête, découvre qu'aucune porte n'est pas forcée et que les deux chiens de garde ne sont pas réveillés. Le songe effrayant du juge et la découverte terrible dans le cimetière, tout un ensemble de faits contribue à créer le climat d'épouvante, d'insolite et d'horreur. D'autre part l'auteur indique une autre possibilité d'interprétation. Le comportement de sir John peut parfois éveiller des soupçons, par exemple: le héros est convaincu que sans la chaîne la main peut toujours s'enfuir. On remarque que cet homme vit dans la crainte constante d'une attaque: il a trois revolvers chargés, ne peut pas dormir et s'enferme chaque soir dans sa chambre. Son passé est voilé, mystérieux. Une fois il dit d'avoir beaucoup chassé, l'homme aussi. Rowell évite les contacts avec son entourage, a le goût pour les choses macabres (la main d'écorché), son comportement peut facilement s'expliquer par la manie de persécution. Maupassant a fait son narrateur M. Bermutier - le juge - c'est-à-dire personne incrédule aux explications surnaturelles. M. Bermutier croit que sir John souffre d'une maladie mentale et c'est pour cette raison que l'Anglais se comporte d'une manière tellement bizarre. Le narrateur soupçonne que le propriétaire de la main coupée est entré pour se venger et c'était lui qui a tué sir John Rowell.

Dans La Main il n'y a que des allusions qui nous suggèrent la maladie mentale du héros. Dans le récit suivant Lui? cette maladie est évidente en tant qu'interprétation rationnelle. Le héros de Lui? c'est un jeune homme - M. Raymon - atteint d'une bizarre phobie: «J'ai peur de moi! J'ai peur de la peur; peur des spasmes de mon esprit qui s'affole, peur de cette horrible sensation de la terreur

\footnotetext{
${ }^{5}$ L. Vax, La sêduction de l'étrange, P.U.F., Paris 1987, p. 27.
} 
incompréhensible» ${ }^{6}$. Un jour, M. Raymon, touché d'une inexplicable tristesse, remarque dans son fauteuil un homme. Mais ce n'est que l'illusion qui se dissipe après quelques instants. Le héros minimise ce fait et tente de l'expliquer par «un accident nerveux de l'appareil optique»" ${ }^{7}$. Mais l'illusion revient en rêve. Le héros sent la présence de l'hôte partout. Maupassant présente d'une manière très suggestive la progression de l'angoisse liée à l'aggravation des symptômes de maladie. Le héros reste hanté par sa propre folie, il peut évoquer tout les détails de son cas, il le fait avec lucidité, mais il n'est pas capable d'échapper à son mal. Juste au commencement du récit, il affirme de ne pas croire aux miracles, ni aux revenants. Il croit à l'anéantissement de chaque être après la mort. Cependant la vision revient. D'une manière très lucide le héros cherche à déterminer les causes de son hallucination, à la fin il tire la conclusion: "Car il est là parce que je suis seul, uniquement parce que je suis seul»". Selon P.-G. Castex l'hallucination du héros a le caractère autoscopique, c'est-à-dire c'est l'hallucination par laquelle on croit se voire soi-même. En fait, le héros accomplit des conditions psychologiques qui favorisent l'autoscopie: c'est la solitude qui a donné naissance à son double. M. Raymon ayant besoin de la présence constante d'une autre personne, veut mettre fin à sa solitude. Dans son hallucination il se voit abandonné, endormi devant la cheminée. Sa vision est la projection de ses angoisses, il a peur d'être seul, d'être abandonné - c'est pourquoi il voit son double lui aussi solitaire. Le titre du récit nous paraît significatif: Lui? Dès 1882 Maupassant connaît de semblables hallucinations, le récit pourrait alors revêtir la dimension autobiographique. Le titre ne serait - il donc qu'un procédé de mystification? P.-G. Castex l'affirme dans Le conte fantastique en France de Nodier à Maupassant (p. 337).

Les années 1884-1886 se distinguent non seulement par le progrès de la maladie nerveuse de Maupassant mais aussi par le retour des thèmes obsédants dans son œuvre. En 1884 il publie La chevelure. Le narrateur du récit visite une maison de santé où l'on lui rend accessible le journal intime d'un fou rongé par sa pensée, atteint de folie érotique et macabre, d'une sorte de nécrophilie. Dans son journal le fou raconte sa vie tranquille et sans amour. Il n'avait qu'une faiblesse: les meubles anciens et les vieux objets. Un jour il a acheté un meuble italien du XVII ${ }^{\mathrm{e}}$ siècle et il y a trouvé une merveilleuse chevelure de femme. Il en est tombé amoureux ou plutôt il s'est épris de la femme à qui appartenait cette chevelure. C'était une sorte de passion obsédante qui le rendait fou. Une nuit il l'a vue telle qu'elle était vivante autrefois. Emporté par la joie suprême il l'a partout promenée comme sa femme. Il a cru de la posséder chaque nuit. Mais on s'est vite rendu compte de sa maladie et on l'a enfermé dans un asile. Ici finit le document. Après la lecture, le narrateur demande au médecin si cette chevelure existe réellement. En la tenant entre les

${ }^{6}$ G. de Maupassant, Contes étranges, Collection - Promesses, Édition d'Antan, Varsovie 1983, p. 88.

${ }_{7}^{7}$ G. de Maupassant, Contes étranges, p. 88.

${ }^{8}$ Ibidem, p. 93. 
mains il ressent une envie comme devant la tentation d'une chose infâme et mystérieuse.

Le point de départ du récit diffère de deux nouvelles précédantes (La Main, Lui?), dans La chevelure l'action commence dans une maison de santé et la folie du héros est attestée par le médecin. La description de l'aspect physique du fou inspire une sorte d'angoisse, de peur devant quelque chose d'insaisissable, d'inexplicable qui l'a tellement exténué: «Le fou [...] nous regardait d'un œil fixe, vague et hanté. Il était fort maigre, avec des joues creuses et des cheveux presque blancs qu'on devinait blanchis en quelques mois ${ }^{9}$. On pressent l'existence d'une force maléfique qui le tue et qu'on ne peut pas anéantir avec des efforts humains: les médecins ne peuvent rien contre cette maladie. Dans le récit fantastique la rencontre avec le phénomène est toujours une force destructrice pour le personnage. Le héros reste marqué par cette rencontre pour toute sa vie, soit par le changement de l'aspect physique (le blanchissement rapide des cheveux, la silhouette courbée etc.) soit par des troubles mentaux, soit par l'un et l'autre. Dans le récit de l'étrangeté psychique le phénomène surnaturel est remplacé par l'hallucination, la vision, le délire qui devient la forme moderne du surnaturel. Selon J. Malrieu, à chaque personnage correspond un tel phénomène, par exemple le héros de La chevelure se complaît dans le passé et son hallucination revêt la forme d'une femme d'autrefois. Malrieu écrit: «Le phénomène n'est plus alors qu'expression concrète, ou la matérialisation des aspirations, des fantasmes ou des angoisses du personnage ${ }^{10}$. Malrieu remarque aussi qu'on ne peut pas échapper à son phénomène qui devient de plus en plus envahissant et progressivement demeure l'unique préoccupation du personnage: «Je ne pouvais plus me passer d'elle, ni rester une heure sans la revoirs" ". Maupassant présente dans La chevelure un cas typique de la monomanie - délire limité à une seule idée, un seul objet. Le héros solitaire laisse souvent errer son imagination. Sa vie est sans émotions. Une phrase nous avertit que quelque chose d'étrange va arriver: «[...] l'amour est venu me trouver d'une incroyable manière [...]» ${ }^{12}$. Grâce à sa découverte dans le vieux meuble le héros tombe amoureux de la femme à qui appartenait la chevelure. Peut-être cette chevelure d'une femme d'autrefois incarne pour lui un amour idéal qu'il cherchait en vain auprès des femmes terrestres. Sans doute, c'est une sorte de nécrophilie qui revêt la forme d'un amour pour une morte revenue parmi les vivants. La conviction du fou de l'apparition de cette femme provoque chez le lecteur le sentiment d' «inquiétante étrangeté» dont parlait Freud: «L'inquiétante étrangeté surgit souvent chaque fois où les limites entre imagination et réalité s'effacent»» ${ }^{13}$. Selon Freud: «La vie psychique du névrosé est dominé par l'exagération de la réalité psychique par

\footnotetext{
${ }^{9}$ Ibidem, p. 107.

${ }^{10}$ J. Malrieu, Le fantastique, Hachette, Paris 1992, p. 99.

${ }^{11} \mathrm{G}$. de Maupassant, Contes étranges, p. 112.

12 Ibidem.

${ }^{13}$ S. Freud, Essais de psychanalyse appliquée (E.P.A.), Gallimard, Paris 1933, p. 78.
} 
rapport à la réalité matérielle» ${ }^{14}$. Le malade ne s'intéresse plus au monde, renonce à rencontrer des gens. Il s'enferme dans son propre monde, celui de rêves, de visions, de fantasmes. Il perd totalement le contact avec son entourage. Les choses primordiales jadis, plus tard lui deviennent égales. C'est le phénomène qui l'attire, qui l'intéresse le plus, qui devient une seule chose importante pour lui. Maupassant montre dans La chevelure la même situation: le héros abandonne ses préoccupations quotidiennes pour s'enfermer dans son monde psychique. Pas à pas, le personnage devient étranger à la société et finalement à soi-même. L'intensité de ses hallucinations, la perte de la capacité du jugement raisonnable, l'aliénation finale du personnage contribuent à créer le climat du fantastique clinique.

En février 1885 Maupassant publie Lettre d'un fou - le récit contenant déjà le germe du Horla (la scène du miroir sans reflet). C'est une lettre supposée écrite par un homme qui sent vaciller sa raison et sollicite l'avis de son médecin sur le récit qu'il lui adresse. Elle expose l'analyse lucide des motifs qui l'on conduit à remarquer l'impuissance des organes humains. Le héros constate que nos seuls moyens de voir et comprendre le monde sont non seulement limités mais en plus trompeurs. La folie devient pour le héros un autre état, une alternative qui lui permet d'observer la petitesse et l'infirmité des sens humains. Le héros remarque: "L'être intérieur, qui constitue le moi, se trouve en contact, au moyen de quelques filets nerveux, avec l'être extérieur qui constitue le monde»" ${ }^{15}$. L'expression «quelques filets nerveux» pour désigner l'ensemble des organes de sens, souligne l'indigence des moyens humains d'appréhension du monde et la disproportion entre «le moi» et «le monde». L'argumentation présentée par le héros vise à démontrer que la connaissance exacte du monde réel est inaccessible à l'homme du fait de ses limites organiques et sa subjectivité. Cette idée alimente un débat traditionnel de la philosophie du XIX ${ }^{\mathrm{e}}$ siècle. (cf. Spencer Premiers principes, Schopenhauer Fondement de la morale).

Lettre d'un fou peut être conçue comme une ébauche, une esquisse du Horla. On y trouve les mêmes hantises: la présence angoissante d'êtres invisibles, de «passant surnaturel», l'angoisse de l'Inconnu, du vide, les jeux de miroirs. La première version du Horla est publié en octobre 1886, la seconde en mai 1887. C'est la seule fois où l'auteur nous présente deux versions d'un texte sous le même titre. La signification du titre préoccupe de nombreux critiques ${ }^{16}$. Le Horla c'est un

${ }^{14}$ Ibidem, p. 139.

${ }^{15} \mathrm{G}$. de Maupassant, Le Horla et autres contes d'angoisse, Flammarion, Paris 1984, p. 38.

16 *Mansay, L'intermédiaire des chercheurs et des curieux 30 VII 1901: «Horla» veut dire "orla», génitif d'(coriol» - aigle en russe; *A. Vial, Maupassant et l'art du roman, Nizet, Paris 1954, p. 242: «Horla» c'est l'anagramme de Lahor - pseudonyme de Cazalis (ami et médecin de Maupassant); *R. Dumesnil, Guy de Maupassant, Armand Colin, Paris 1933, p. 32. P.-G. Castex, Le conte fantastique... et M.-C. B ancquart dans l'introduction pour Le Horla et autres contes cruels et fantastiques de Maupassant, Classique Garnier, Paris 1976, p. 35 optent pour le mot «horzain» ou "horsan" c'est-à-dire étranger dans le patois normand; *M.-C. Bancquart in ibidem prétend aussi que "Horla» peut provenir de «Horlaville» - le patronyme fréquent en Normandie; ${ }^{2}$. Forestier, Contes 
persécuteur invisible et omniprésent qui investit l'espace psychique du héros, c'est l'entité invisible qui en absorbant le reflet du personnage lui dérobe sa personnalité. Dans la première version du récit le héros anonyme raconte son histoire aux trois aliénistes et aux quatre savants naturalistes. Les paroles du narrateur sont soumises à une sorte d'épreuve de vérité, c'est le docteur Marrande qui se porte garant des affirmations de son patient, l'aliéniste est son avocat, il le défend et lui apporte la caution de son autorité. Le narrateur vivait seul avec ses domestiques dans une maison près de Rouen. Il s'est soudainement senti pris de malaises inexplicables qui s'amplifiaient chaque jour. Son cocher, lui aussi, a été atteint de la même maladie. Peu à peu, le personnage éprouvait le sentiment d'une présence invisible à ses côtés qui se nourrissait littéralement de lui. Les différentes expériences auxquelles il se livrait pour déterminer la nature de son mal n'aboutissaient à rien de décisif, mais le sentiment qu'il éprouvait d'être possédé n'a pas cessé de s'accroître. Un de ses voisins se trouvait dans le même état. Avec la venue de l'hiver les malaises du narrateur ont disparu. Au commencement du printemps l'accident avec la rose cueillie par la main invisible a amené le narrateur à supposer qu'une race d'êtres invisibles et supérieurs, auxquels il a donné le nom de Horla, était en train de s'emparer de l'humanité toute entière. Le narrateur a prié de le garder dans un asile.

La première version c'est le récit a posteriori. La version de 1887 (seconde) est rédigée sous forme de journal intime tenu au présent, le lecteur reste dans l'ignorance de ce qui va advenir. Les séquences sont presque les mêmes et présentées dans le même ordre dans les deux versions ${ }^{17}$. Les dénouements sont cependant différents: quant à la première version le héros se fait garder dans une maison de santé, le récit aboutit finalement à une conclusion faisant appel à la théorie évolutionniste de Darwin et à la science-fiction - genre nouveau qui apparaîtra plus tard. Dans la version de 1887 le héros décide de tuer le Horla en mettant le feu à sa maison dans laquelle il pense avoir enfermé l'être invisible. Mais le héros ne provoque que la mort de ses domestiques et se sent de nouveau possédé. Le manuscrit s'arrête sur sa décision de se suicider. La première version met l'accent sur la présence réelle du Horla dans le monde. J. Van Herp voit le Horla comme «une des premières apparitions du mutant» ${ }^{18}$. Selon $\mathrm{M}$. Dentan la première version du Horla n'est pas un récit fantastique mais «un récit de science-fiction [...] allant un peu au-delà des inventions de Jules Verne») ${ }^{19}$. La première version pourrait être conçue comme «merveilleux scientifique». Le Horla y est le successeur de l'homme, un mutant ou l'extra-terrestre. Cette version tend une certaine objectivation dans la présentation des événements. La seconde version, grâce à sa

et nouvelles de Maupassant, t. II, la Pléiade, Gallimard, Paris 1974-1979, p. 1621 suppose que «Le Horla» c'est l'anagramme de "choléra». Il retient aussi l'interprétation plus simple - «hors là».

${ }^{17}$ Les épisodes tels que le voyage au Mont Saint-Michel, à Paris, l'épisode avec le magnétisme ne sont présents que dans la seconde version.

18 J. van Herp, Fantastique et mythologies modernes, Édition Rectol. Verso, Bruxelles 1985 , p. 48.

${ }^{19}$ M. Dentan, Le Horla ou le vertige de l'absence, in: Études de lettres, Lausanne 1976, p. 50. 
forme du journal intime, met le lecteur en contact direct avec l'interprétation des événements par le prétendu fou. Pour le narrateur son journal devient une sorte de garde-fou. Le héros relate des événements, les commente mais on ne peut pas être sûr de la véracité de son histoire. Durant tout le récit il s'interroge sur son éventuelle folie.

Étant donné le sujet de cet article nous prendrons en considération la seconde version où l'interprétation liée aux troubles mentaux du héros est plus convaincante.

Le Horla dans la seconde version peut être conçu comme le vampire mental, voire psychique. Sous la date du 4 juillet le héros avoue dans son journal qu'une nuit il a senti quelqu'un accroupi sur lui et qui puisait dans sa gorge et buvait sa vie. Cette sensation n'est pas encore liée par le narrateur à l'existence d'un être qui en serait la cause, elle évoque les hallucinations associées au vampirisme dans certains traités du XIX ${ }^{\mathfrak{e}}$ siècle, comme par exemple le traité De la folie de 1845 écrit par L.-F. Calmeil. Le vampirisme s'y trouve défini comme une «monomanie fondée sur des hallucinations de la vue et du toucher survenues le plus souvent pendant le sommeil et susceptibles d'affecter un certain nombre de sujets appartenants à la même famille ou à la même contrée» ${ }^{20}$. Les sujets hallucinés se sentaient comme «étreints par les bras du même revenant» ${ }^{21}$ et croyaient qu'une «bouche collée sur quelque partie de leur corps y suçait le sang avec avidité» ${ }^{22}$. L'image de la sangsue est ici appelée par l'idée d'un être qui suce la vie comme le vampire qui suce le liquide vital - le sang. Le Horla pourrait donc être conçu comme la métamorphose de la maladie, de la folie qui ronge l'homme, qui l'épuise, qui le dévore, qui cause enfin son échec. On peut aussi voir dans le Horla - le double du héros. Le 13 août le narrateur note: «Quelqu'un possède mon âme et la gouverne! Quelqu'un ordonne tous mes actes, tous mes mouvements, toutes mes pensées. Je ne suis plus rien en moi...» ${ }^{23}$. Le héros est hanté par la pensée qu'une part de soi prendra le contrôle de l'ensemble de sa psychique dont le résultat sera l'aliénation et la folie. Maupassant lui aussi a été victime de l'hallucination autoscopique et du dédoublement. L'homme est double dès l'origine, il est partagé entre le bien et le mal. Quand cette dualité se concrétise, le double s'incarne et tente de se substituer au héros. Dans Le Horla le double du héros devient autonome, il ne lui suffit plus d'investir l'espace psychique du narrateur, le Horla est venu pour vampiriser toute l'humanité. $R$. Ruyer remarque que le double est «avant-coureur de la mort ou de la folie, mort spirituelle» ${ }^{24}$. Le héros du Horla accomplit du point de vue psychologique les conditions favorables pour la folie: il aime la solitude, il faut noter aussi l'absence de parents proches (sauf la cousine parisienne) et l'absence de présence féminine. Dès le commencement les phases de la joie, de l'excitation et de la tristesse, de l'anxiété alternent chez le héros ce qui est très caractéristique pour les maladies

\footnotetext{
${ }^{20}$ L.-F. Calmeil, De la folie, Baillière, Paris 1845, p. 54.

${ }^{21}$ Ibidem.

${ }^{22}$ Ibidem.

${ }^{23}$ G. de Maupassant, Le Horla et autres..., p. 79.

${ }^{24} \mathrm{R}$. Ruyer, La nature du psychique, Revue de métaphysique et de morale, 1952, no 1, p. 46.
} 
mentales (le 8 mai: «Quelle journée admirable! [...] J'aime ce pays et j'aime y vivre [...]»; le 12 mai: «Je me sens souffrant, ou plutôt je me sens triste $[\ldots]{ }^{25}$ ). Maupassant décrit d'une manière détaillée les hallucinations dont son héros est la victime, entre autres l'hallucination négative - le héros n'aperçoit pas son reflet dans la glace et l'hallucination visuelle - le héros voit la rose cueillie par la main invisible. L'hallucination occupe une place importante parmi les symptômes de la folie. Maupassant tient à la vraisemblance psychologique de son héros. L'auteur inscrit son récit dans le cadre réaliste du XIX ${ }^{\mathrm{e}}$ siècle, il essaye d'éveiller des doutés chez le lecteur: les événements relatés seraient - ils seulement le fait de la conscience affolée? D'une telle manière naît le fantastique psychique qui fait partie de la réalité la plus quotidienne et la plus banale.

Moiron publié en 1887 c'est une histoire effrayante de l'instituteur Moiron atteint d'une folie infanticide. Il a eu trois enfants, morts successivement de la poitrine. Il semblait qu'à partir de ce moment-là Moiron avait encore plus de tendresse pour les enfants de sa classe. Il achetait des bonbons et des gâteaux pour ses meilleurs élèves. Mais soudain cinq de ses élèves sont morts d'une étrange et mystérieuse maladie. On a cherché des causes mais on a rien découvert. Après un an encore deux élèves ont expiré d'une façon bizarre. Grâce à l'examen des corps on a découvert des fragments de verre pilé incrustés dans les organes. Personne ne soupçonnait Moiron jusqu'au jour où la servante de l'instituteur est tombée malade après avoir mangé des bonbons achetés par Moiron pour ses élèves. L'instituteur a été arrêté, on a accumulé des preuves tangibles contre lui, pourtant on ne voyait aucun motif qui pouvait pousser Moiron aux crimes tellement odieux. Il a été condamné à mort. Pendant la confession, Moiron a avoué au prêtre d'être innocent. La peine de mort a été commuée en celle des travaux forcés. Après quelques années Moiron, grâce à sa conduite exemplaire, a été employé comme domestique dans l'établissement pénitencier. Avant sa mort Moiron a avoué toute la vérité au magistrat M. Malaureau. L'instituteur a exposé les motifs qui l'on porté aux crimes: après la mort de ses enfants il voulait se venger contre Dieu injuste et méchant. Tout être a pour mission de tuer, chacun en est capable, tout être a alors une prédisposition à la folie qui est le réveil de la nature criminelle de l'homme - telle est la philosophie de Moiron. Il est aussi en quelque sorte jaloux de pouvoirs destructeurs de Dieu, l'instituteur déclare avoir tué les enfants pour devenir égal de Dieu qui a tué les siens. En tuant Moiron veut dérober la puissance divine et il y prend plaisir d'une nature prométhéenne: «Ce n'est pas lui qui les a eus, ceux-là Ce n'est pas lui, c'est moi» ${ }^{26}$. Bien que Moiron soit qualifié de dément, il analyse les crimes commis avec une certaine lucidité. D'habitude on souligne que le crime commis dans un moment de folie a pour circonstance atténuante l'absence de préméditation. Mais le cas de Moiron est différent: il nous semble que les fous sont aussi capables de préméditation que les gens sains d'esprit. Moiron perpètre des

${ }^{25}$ G. de Maupassant, Le Horla et autres..., p. 55.

${ }^{26}$ G. de Maupassant, La petite Roque et autres histoires criminelles, Flammarion, Paris 1989 , p. 234. 
séries d'assassinats sachant que sa profession le met à l'abri du soupçon. En plus il ment au prêtre pendant la confession pour se sauver. Avec Moiron Maupassant nous donne l'analyse approfondie de la folie qui a transformé un homme pieux et bon en bête qui trouve son unique plaisir dans le meurtre. Ce récit fantastique de l'étrangeté psychique est fondé sur la présentation du cas de l'obsession morbide poussant aux crimes. L'univers mental du héros est un monde vraiment fantastique, étrange et étranger au lecteur. La folie y devient mystérieuse car elle peut tout: changer complètement la psychique humaine, atteindre chacun - même celui qui paraît être le plus sain d'esprit, enfin pousser aux actes les plus terribles. Les conséquences de la démence y présentées sont néfastes. La folie du héros a une inquiétante et irréductible étrangeté.

Le récit suivant, Madame Hermet, a été publié en 1887 après la visite de Maupassant dans un asile d'aliénés à Tunis. Le narrateur du récit visite aussi la maison de santé. On lui montre un cas intéressant: une femme d'environ quarante ans, encore belle, qui regarde tout le temps son visage dans la glace. Après avoir aperçu les gens, elle s'enveloppe d'une voile pour cacher son visage. Elle se plaint au médecin d'être laide, défigurée à cause des marques qui augmentent tous les jours. Ensuite elle explique d'avoir contracté cette maladie en soignant son fils. Le médecin l'entraîne à découvrir son visage. Le narrateur constate avec étonnement qu'elle n'a rien sur la figure, pas une marque. Plus tard le médecin lui raconte l'histoire de Madame Hermet. Elle était très belle, heureuse de vivre, elle aimait les divertissements mondains. Mais le temps passait et elle vieillissait. Chaque jour elle regardait son visage dans la glace en cherchant des rides. Un jour son fils est tombé malade d'une petite vérole. Avant sa mort il désirait voir sa mère. Madame Hermet avait tellement peur de la contagion qu'elle n'a pas satisfait la demande du mourant. Quand son fils est mort, elle est devenue folle.

Le cas de la folie de Madame Hermet est différent des autres présentés dans les récits de Maupassant: la folie de Madame Hermet a les raisons concrètes. L'héroïne devient folle à cause des remords qu'elle éprouve après avoir abandonné lâchement son fils mourant. G. Ponnau décrit le syndrome du fantastique qui «consiste à se punir d'un crime par des visions terrifiantes» ${ }^{27}$. Madame Hermet est la victime de ce syndrome. Elle a commis le crime en refusant d'assister son fils mourant. Devenue folle est se punit elle-même par la conviction qu'elle est laide. Sa beauté était la plus importante pour elle et à causes des hallucinations Madame Hermet est convaincue d'être à jamais défigurée. Elle croit qu'un dieu sadique se venge d'elle en couvrant son visage de trous abominables et en la rendant par cela repoussante. Certains symptômes du mal qui s'approche sont visibles même avant la maladie du fils de Madame Hermet. L'héroïne passe des heures et des heures devant la glace, elle cherche des rides, elle a peur de perdre sa beauté, elle redoute la vieillesse. Son lâche comportement envers son fils a le rôle d'un catalyseur: à cause des hallucinations visuelles ses angoisses sont extériorisées. La folie devient la

${ }^{27}$ G. Ponnau, La folie ..., p. 165. 
projection des hantises et des obsessions du personnage. Dans ce récit il n'y a pas d'éléments fantastiques de convention, il n'y a pas de surnaturel traditionnel. Le récit fantastique de l'étrangeté psychique repose sur des données cliniques: le surnaturel se trouve dans l'esprit ébranlé de l'héroïne - prisonnière de ses effroyables visions. L'esprit humain paraît ếtre capable de tout, c'est un lieu mystérieux et effrayant et c'est aussi le domaine le plus exploré dans le fantastique psychique.

Qui sait? est le dernier récit fantastique de Maupassant (de 6 IV 1890). De retour chez lui après une sortie nocturne, le narrateur - personnage a la surprise de voir ses meubles et ses objets qui constituent son seul attachement sur terre, s'enfuir sous ses yeux, sans que ses efforts pour les rattraper aboutissent à quoi que ce soit. Le narrateur se croyant malade fait le voyage de quelques mois. Passant par Rouen il aperçoit dans la boutique d'un étrange brocanteur tous les meubles qui étaient partis de chez lui. Il prévient la police mais les meubles et le brocanteur disparaissent entre-temps. Nouveau rebondissement: quand le héros est informé par une lettre de son domestique que meubles et objets sont revenus dans la maison aussi soudainement qu'ils en étaient partis. Pour échapper à une éventuelle rencontre qu'il redoute avec le mystérieux brocanteur, le narrateur demande son internement volontaire dans un asile, tout en vivant dans la hantise de voir l'autre apparaître dans le pavillon où il a choisi de se faire enfermer. Au commencement du récit le héros - narrateur écrit son journal enfermé volontairement dans une maison de santé. Avant la lecture de tout le récit le lecteur peut admettre qu'il s'agit de l'histoire du malade mental. Les traits caractéristiques du héros révèlent une sensibilité pathologique: c'est un type de rêveur solitaire, de philosophe isolé, les gens le lassent et ennuient, il les fuit pour vivre dans son monde intérieur: «Je me fatigue très vite de tout ce qui ne se passe en moi» ${ }^{28}$. Un tel repliement sur soi est caractéristique pour les personnages fantastiques. J. Malrieu dans Le fantastique (pp. 56-59) distingue trois types de l'isolement du héros fantastique: l'isolement social, affectif et intellectuel. Le héros de Qui sait? s'isole volontairement de la société: sa maison à la compagne est loin de la route, le héros n'a pas de voisins. On remarque aussi chez lui l'isolement affectif: ce sont des objets qui prennent pour le héros l'importance d'êtres. Le personnage est célibataire, il préfère vivre entouré des bibelots, de vieux meubles. Quant à l'isolement intellectuel - on l'observe aussi chez lui. Le héros est un homme cultivé qui se sent supérieur aux autres. C'est un philosophe qui ne sait plus communiquer avec autrui. Comme les gens l'ennuient et l'énervent, il crée son propre monde où il croit vivre heureusement. L'étiologie de la folie du héros paraît inconnu mais on sait que pour le narrateur tous ses objets étaient chers et importants, inconsciemment il avait peur de les perdre. Les hallucinations ne seraient que la projection de ses angoisses. Malrieu écrit: «On a le phénomène que l'on est» ${ }^{29}$. C'est pourquoi le héros attaché à ses objets assiste

${ }^{28}$ G. de Maupassant, Contes et nouvelles, t. II, Nouveaux Classiques Larousse, Paris 1974, p. 116.

${ }^{29} \mathrm{~J}$. Malrieu, Le fantastique, p. 105. 
impuissant dans sa vision à la fuite de son mobilier. Le héros agit avec une bizarre logique: il ne veut pas acheter d'autres meubles - «Mais je ne remeublai pas ma maison. C'était bien inutile. Cela aurait recommencé toujours» ${ }^{30}$. Le narrateur se croit persécuté à l'éternité par des forces inconnues. Aussi le personnage du brocanteur l'inquiète beaucoup, le brocanteur est pour lui l'incarnation des forces maléfiques. L.Vax signale le brocanteur comme personnage inquiétant dans les récits fantastiques: «[...] l'antiquaire [...] ne vit pas seulement dans le présent mais participe en quelque sorte au passé parmi les choses vieilles qu'il achète et qu'il vend. Il est toujours vieux, sa boutique est profonde, obscure et poussiéreuses ${ }^{31}$. À la fin du récit redoutant la prétendue vengeance du brocanteur, le héros se fait interner dans la maison de santé. Ce comportement se traduit par la paranoïa (à force des hallucinations le malade sort du monde réel, il a les tendances pour la fausseté du jugement, la méfiance, son sens autocritique est perturbé, souvent il se croit persécuté).

Qui sait? est le dernier récit fantastique de Maupassant et aussi le dernier récit fantastique de l'étrangeté psychique que nous voudrions analyser dans la présente étude. Les récits de l'étrangeté psychique sont la forme moderne du surnaturel. Les cuvres du fantastique clinique «s'apparentent aux peintures d'aliénés et aux obsessions d'alcooliques ou des dégénérés, le sadomasochisme y jouant un rôle important ${ }^{32}$. Chez Maupassant le centre de l'univers fantastique est le cerveau du malade. Ce sont les visions, les hallucinations, les obsessions du héros qui provoquent la peur chez le lecteur. Mais cette peur est spécifique. Ce n'est pas la peur primitive et superstitieuse devant les fantômes, les diables, les vampires. C'est l'angoisse devant l'Inconnu qui est en nous-mêmes. L'homme a peur de la puissance de son esprit. Le danger qui hante le héros est intériorisé et par cela plus inquiétant. La folie, le clinique remplacent le phénomène surnaturel traditionnel. Les descriptions de l'état mental du héros, de son aspect physique, de son comportement créent le climat d'épouvante moderne. Dans les récits de l'étrangeté psychique c'est l'aspect psychotique qui prend du relief chez Maupassant. On retrouve toujours la réalité banale et quotidienne d'une ville française du XIX siècle comme le cadre pour les événements relatés. Si cette réalité est d'abord rassurante, c'est pour mieux montrer la brusque émergence du fantastique dans le monde réel. Depuis ce moment-là dans le récit de l'étrangeté psychique la frontière entre le réel et le surnaturel devient floue. On n'est jamais capable de définir la nature du phénomène: tout peut s'expliquer par des troubles mentaux du héros mais l'intervention du phénomène peut aussi revêtir le caractère sumaturel. L'aventure insolite ne peut être qu'une vision mais aussi bien elle peut avoir lieu en réalité. Le lecteur hésite toujours car l'auteur de sa part n'explique rien.

G. de Maupassant est un des plus connus auteurs de récits fantastiques du XIX ${ }^{\mathrm{e}}$ siècle. Son intérêt pour le fantastique clinique est surtout visible dans ses récits de

${ }^{30} \mathrm{G}$. de Maupassant, Contes et nouvelles, t. II, p. 119.

${ }^{31}$ L. V ax, Art et littérature fantastiques, P.U.F., Paris 1970, p. 34

${ }^{32}$ J.Z. B ernard, Dictionnaire de l'insolite..., p. 119. 
l'étrangeté psychique. Cependant on retrouve les traits du fantastique d'ordre psychique chez Maupassant dans les récits sur les phénomène parapsychologiques et dans les histoires renouvelées de rêves, de paradis artificiels, de visions. Nous sommes conscients que pour mieux montrer la complexité du fantastique clinique de Maupassant il serait indispensable de parler encore de relations entre ces deux types de récits fantastiques et la folie. Pourtant une étude si poussée dépasserait le cadre du présent article. Nous tâcherons par la suite de revenir à ces problèmes-là.

\section{BIBLIOGRAPHIE}

B ancquart M.-C. (1976), Le Horla et autres contes cruels et fantastiques de Maupassant, Classique Garnier, Paris, pp. 357.

Bernard J.-L. (1977), Dictionnaire de l'insolite et du fantastique, Éditions du Dauphin, Paris, pp. 418.

Calmeil L.-F. (1845), De la folie, Baillière, Paris, pp. 500.

Castex P.-G. (1994), Le conte fantastique en France de Nodier à Maupassant, José Corti, Paris, pp. 466 .

Dentan M. (1976), Le Horla ou le vertige de l'absence in: Études de lettres, Lausanne, pp. 108.

Dumesnil R. (1933), Guy de Maupassant, Armand Colin, Paris, pp. 246.

Forestier L. (1974-1979), Contes et nouvelles de Maupassant, t. II, La Pléiade, Gallimard, Paris, pp. 1766.

Freud S. (1933), Essais de psychanalyse appliquée, (E.P.A.) Gallimard, Paris, pp. 279.

Herp J. van (1985), Fantastique et mythologies modernes, Édition Rectol. Verso, Bruxelles, pp. 429.

Malrieu J. (1992), Le fantastique, Hachette, Paris, pp. 160.

Mans ay (1901 VII 30), L'intermédiaire des chercheurs et des curieux.

Maupassant G. de (1974), Contes et nouvelles, t. II, Nouveaux Classiques Larousse, Paris, pp. 159.

Maupassant G. de (1983), Contes étranges, Collections - Promesses, Éditions d'Antan, Varsovie, pp. 286.

Maupass ant G. de (1984), Le Horla et autres contes d'angoisse, Flammarion, Paris, pp. 254.

Maupassant G. de (1989), La petite Roque et autres histoires criminelles. Flammarion, Paris, pp. 316.

Ponnau G. (1987), La folie dans la littérature fantastique, CNRS, Paris, pp. 355.

Ruyer R. (1952), La nature du psychique in: Revue de métaphysique et de morale, $\mathrm{n}^{\circ} 1$, pp. 252.

Vax L. (1970), Art et littérature fantastiques, Presses Universitaires de France, Paris, pp. 128.

Vax L. (1987), La séduction de l'étrange, Presses Universitaires de France, Paris, pp. 313.

Vial A. (1954), Maupassant et l'art du roman, Nizet, Paris, pp. 268. 